\title{
Aprendizaje colaborativo mediado por TIC en una escuela pública con niñez migrante de Montevideo
}

Cultura(s) en clave de extensión universitaria / Intervenciones

\author{
Mónica Da Silva Ramos \\ mdasilva@psico.edu.uy \\ (iD) orcid.org/0000-0003-2522-4716

\section{Estela Rivero Vidal} \\ estelariverovida/@gmail.com \\ (iD) orcid.org/0000-0002-8709-6572 \\ Instituto de Fundamentos y Métodos \\ en Psicología de la Universidad de la \\ República, Uruguay.
}

RECEPCIÓN: 24/06/19

ACEPTACIÓN FINAL: 30/10/19

\section{Resumen}

Este artículo parte de un proyecto de extensión universitaria que diseñó e implementó una propuesta de aprendizaje colaborativo a través del uso de Tecnologías de la Información y Comunicación (TIC), en una escuela con niñez migrante en la ciudad de Montevideo, con el fin de generar procesos de inclusión intercultural. Se utilizó una metodología cualitativa, basada en el diseño de un modelo colaborativo y narrativo, con entrevistas, registros etnográficos y una escala de motivación y de observación de clases. Se presentan algunos resultados de dicho proyecto con el fin de problematizar algunas dimensiones analíticas, como las características del diseño colaborativo y su impacto en los participantes, la creación de una narrativa colaborativa como hilo conductor de la propuesta y el papel mediador de las TIC como elementos significativos para la generación de un espacio de respeto a la diversidad. Las reflexiones se enfocan en el papel de la construcción de propuestas situadas, basadas en la identificación de los fondos de conocimiento de las familias y niñez migrantes.

Palabras clave: colaboración, interculturalidad, Tecnologías de la Información y la Comunicación, Modelo Quinta Dimensión.

\author{
Nicolás Chiarino Durante \\ nchiarino@psico.edu.uy \\ (iD) orcid.org/0000-0002-4530-1777
}

\author{
Luis Osvaldo Pereyra Elordi \\ Ipereyra@psico.edu.uy \\ (iD) orcid.org/0000-0001-9703-6138
}

Instituto de Psicología, Educación y Desarrollo Humano de la Facultad de Psicología de la Universidad de la República.
Aprendizagem colaborativa mediada pelas TIC em uma escola pública com crianças migrantes em Montevidéu

\section{Resumo}

\section{Abstract}

This article starts from a university extension project that designed and implemented a collaborative proposal that integrated the use of Information and Communication Technologies (ICT), in a school with migrant children in the city of Montevideo, in order to generate processes of intercultural inclusion. A qualitative methodology was used, based on the design of a collaborative and narrative model, using interviews, ethnographic records, a motivation scale and class observation. Some results of this project are presented, in order to problematize some analytical dimensions such as the characteristics of the collaborative design and its impact on the participants, the creation of a collaborative narrative as the guiding thread of the proposal and the mediating role of ICT as significant elements for the generation of a space of respect for diversity. The reflections focus on the role of constructing situated proposals, based on the identification of knowledge funds of migrant and non-migrant families and children.

Keywords: collaboration, interculturality, information and communication technologies, Model Fifth Dimension.
Este artigo parte de um projeto de extensão universitária que implementou uma proposta colaborativa que integrou o uso das Tecnologias da Informação e Comunicação (TIC), em uma escola com crianças migrantes na cidade de Montevidéu, a fim de gerar processos de inclusão intercultural. Utilizouse uma metodologia qualitativa, baseada no desenho de um modelo colaborativo $e$ narrativo, utilizando entrevistas, registros etnográficos, escala de motivação e observação de aula. São apresentados alguns resultados deste projeto, a fim de problematizar algumas dimensões analíticas, como as características do design colaborativo e seu impacto sobre os participantes, a criação de uma narrativa colaborativa como fio condutor da proposta e o papel mediador das TIC como elementos significativos para a geração de um espaço de respeito à diversidade. As reflexões enfocam o papel da construção de propostas situadas, baseadas na identificação de fundos de conhecimento de famílias e crianças migrantes e não migrantes.

Palavras-chave: colaboração, interculturalidade, tecnologias da informação e comunicação, Modelo Quinta Dimensão.

Para citación de este artículo: Da Silva Ramos, M.; Rivero Vidal, E.; Chiarino Durante, C. y Pereyra Elordi, L.O. (2019). Aprendizaje colaborativo mediado por TIC en una escuela pública con niñez migrante de Montevideo. +E: Revista de Extensión Universitaria, 9(11), 130-150. doi: 10.14409/ extension.v9i11.Jul-Dic.8718. 


\section{Introducción}

La movilidad humana es un tema prioritario en la agenda mundial, lo cual requiere que los Estados revisen, diseñen e implementen políticas migratorias basadas en las garantías de los derechos humanos. Uruguay siempre fue un país con movimientos migratorios, pero en los últimos años es receptor de un nuevo flujo migratorio sur-sur, personas de países latinoamericanos de migración no tradicional para nuestra sociedad, tales como: Cuba, República Dominicana, Perú, Venezuela, Colombia, Haití, además de los tradicionales, Argentina y Brasil (Uriarte, y Urruzola, 2018). Por otro lado, también está llegando gran número de personas provenientes de diferentes países de África. Este movimiento migratorio viene siendo fuertemente "racializado", discriminado y "marcado" por gran parte de la sociedad uruguaya, en especial por el rechazo de sus características fenotípicas, niveles educativos y miedos por competitividad en el mercado laboral (Koolhaas, Prieto, y Robaina, 2017). Gran parte de esta población se desplaza por dificultades socioeconómicas y se encuentra en situación de vulnerabilidad social, lo que conlleva, entre otros factores, a dificultades en el acceso al mercado laboral, a la vivienda, a la salud y a la educación.

La inserción en la nueva sociedad sitúa a la población migrante ante procesos socioculturales complejos y ambivalentes, que implican desarraigo y renuncia en parte a la adscripción de su identidad de origen. Ello requiere cambios profundos, flexibilidad para el aprendizaje e incorporación de un nuevo lenguaje y/o estilos de habla, de otras costumbres, lógicas institucionales, nuevas formas de interacción con la población nativa, así como la incorporación de conocimientos que son requeridos en la sociedad de destino. En este sentido, la niñez migrante, en la mayoría de los casos, sin tener una participación real en la decisión de migrar, se ve alejada de su cultura y modos de enculturación, de sus contextos de socialización cotidianos e institucionales, de sus afectos más cercanos: familiares, compañeros/as de aula, amigos y hasta de sus mascotas; de sus hogares, de sus hábitos y geografías, de su clima, de su estilo de habla y/o lengua, de los diversos ámbitos de integración en sus países de origen, tal como la educación, que es uno de los derechos fundamentales que se deben garantizar (Da Silva, y Rivero, 2018). Asimismo, deben aprender e interiorizar una nueva cultura y formas de socialización sin contar muchas veces con los dispositivos necesarios y el acompañamiento adecuado a nivel afectivo e institucional para ello en el país en el cual pasan a residir, ya que su referente adulto está casi completamente dedicado a la búsqueda de trabajo formal.

Respecto de la inclusión en el sistema educativo, Debandi (2018) plantea que hay una ausencia de reconocimiento de los saberes previos de los niños y niñas migrantes en la mayoría de los centros escolares, lo que genera situaciones de discriminación y exclusión que incrementan sus vulnerabilidades y que se ve reflejado en su integración educativa.

Entendemos la interculturalidad desde una perspectiva crítica, como noción que nos permite comprender el encuentro de personas provenientes de distintas culturas en un mismo territorio nacional, pero reconociendo las relaciones de poder, racialización y discriminación que han sufrido históricamente las poblaciones que se han tenido que desplazar desde sus lugares de origen; estas se expresan de manera cotidiana en las interrelaciones que van produciendo y en sus procesos singulares de "adaptación" (nunca total) a las normas e instituciones de la sociedad receptora, necesaria para su participación en la misma (Walsh, 2009). Desde esta perspectiva, pensamos el trabajo con la niñez como una apuesta a la diversidad y a la interculturalidad, como forma de re-existencia en el aula, promoviendo encuentros 
de reconocimientos desde las diferencias, la potencia intersubjetiva y los diversos fondos de conocimientos (Moll, 1997), en un marco de enriquecimiento mutuo y de respeto a los derechos humanos. Los fondos de conocimiento son: "Los cuerpos de conocimiento culturalmente desarrollados e históricamente acumulados, así como las habilidades esenciales para el funcionamiento y bienestar familiar o individual" (Moll:47).

Este artículo surge a partir de reflexiones en cuanto al proyecto de extensión desarrollado desde el Instituto de Fundamentos y Métodos en Psicología de la Facultad de Psicología de la Universidad de la República Oriental del Uruguay (UdelaR) denominado "Construyendo ciudadanía digital mediante la creación de un espacio intercultural en la Ciudad Vieja de Montevideo". El trabajo desde esta experiencia de extensión se centró en la capacitación y uso no instrumental en TIC con niños y niñas procedentes de países de la región en el sistema educativo uruguayo, inspirado en el Modelo colaborativo Quinta Dimensión (en adelante 5D) diseñado especialmente por Michael Cole (2006) para la integración de la niñez migrante.

Se exponen aquí la experiencia de extensión, el procedimiento de intervención llevado adelante, algunos resultados que nos hacen cuestionar la diversidad en las aulas y los procesos de interculturalidad.

A continuación, se presentan las características generales del proyecto, se abordan brevemente el marco teórico que lo sustenta, la metodología y técnicas utilizadas como modo de ubicar al lector en la experiencia de extensión desarrollada. Luego se muestran algunos resultados analizados desde la perspectiva de los procesos de interculturalidad y extensión crítica. Para finalizar, se arriba a algunas reflexiones que buscan generar nuevas líneas analíticas.

\section{Características del proyecto de extensión}

Este proyecto surge de la demanda de la Organización de la Sociedad Civil (OSC) Idas y Vueltas, con larga trayectoria de trabajo con población migrante e incidencia política sobre la situación de vulneración de derechos humanos en el tema. También de centros educativos ubicados en la zona del Municipio B de la ciudad de Montevideo. A partir de ello, realizamos una indagación en campo sobre las necesidades y dificultades de migrantes recién arribados al país en situación de vulneración de derechos. Tuvimos especialmente en cuenta en su diseño el abordar una problemática planteada por los propios actores contactados como urgente, a la vez que analizamos la viabilidad para su implementación de acuerdo con los requisitos planteados en el llamado de la Comisión Sectorial de Extensión y Actividades en el Medio (CSEAM) de la UdelaR.

El proyecto tuvo como objetivo construir un espacio intercultural y participativo en un centro de educación formal que permitiera la generación de procesos de ciudadanía digital.

La elección de la escuela para el trabajo respondió a tres criterios: ser la segunda escuela de Montevideo con mayor número de niños y niñas migrantes, no tener intervención de otros proyectos universitarios ni comunitarios, y estar ubicada en la zona céntrica, en donde se encuentra, junto a Ciudad Vieja y La Aguada, la gran mayoría de migrantes recién arribados a Uruguay en situación de vulneración de social. El desarrollo de esta experiencia, que se llevó adelante durante el año 2018, se acordó conjuntamente con el equipo de Dirección escolar una vez aprobada la propuesta, conforme a las necesidades detectadas por los actores educativos de la escuela, quienes nos asignaron un aula en la que la mitad 
de su población estaba conformada por niños y niñas recién llegados al país que ingresaban a la escuela pública.

El equipo estuvo integrado por cinco docentes universitarios pertenecientes al Instituto de Fundamentos y Métodos en Psicología y al Instituto de Psicología, Educación y Desarrollo Humano de la Facultad de Psicología de la UdelaR; dos psicólogos con formación en tecnología educativa, una psicóloga con formación en educación y una antropóloga con experiencia de trabajo en migración, e integró estudiantes universitarios de la carrera de Licenciatura en Psicología en una modalidad de pasantía de extensión universitaria.

La propuesta estuvo dirigida a 22 niñas y niños uruguayos y migrantes provenientes de Perú, Cuba, República Dominicana y Venezuela, de un aula escolar de $3^{\circ}$ grado. Participaron también los referentes familiares de dicho grupo escolar a través de reuniones con el equipo de docentes, estudiantes y la maestra del aula.

El equipo de Dirección de la escuela, con el cual mantuvimos múltiples reuniones para detectar la demanda específica de este centro educativo, fue quien aprobó, monitoreó y realizó una evaluación final del proyecto integrada al Informe Anual 2018 presentado al Consejo de Educación Inicial y Primaria (CEIP).

La maestra tuvo un rol muy activo: desde la habilitación del espacio para el trabajo del equipo y los estudiantes universitarios en el aula así como su presencia en las reuniones con referentes familiares, su participación en las reuniones de evaluación de cada actividad junto a los estudiantes universitarios y al equipo docente, y la asunción del papel de observadora de cada subgrupo de niños y niñas, según una escala brindada por el equipo docente, durante algunas de las actividades.

Asimismo, fueron nueve los estudiantes de la Licenciatura en Psicología de la UdelaR que participaron de este proyecto de extensión mediante la modalidad curricular de integración a proyectos del Ciclo de Formación Integral.

En las reuniones mantenidas con el equipo de Dirección de la escuela, con la maestra del aula y con los familiares de los niños y niñas, se recabó información sobre las situaciones planteadas como problemáticas y/o sin propuestas de abordaje específicas:

- Alto número de niños y niñas extranjeros en el aula procedentes de diferentes nacionalidades, recién arribados al país: Cuba, Venezuela, Perú y República Dominicana.

- Presencia de niños y niñas con diferentes situaciones problemáticas para la escuela: un niño con dificultades severas de aprendizaje; un niño diagnosticado con trastorno del espectro autista; niños y niñas que vivían en Centros de Acogida del Estado; niños provenientes de colegios privados, así como varias situaciones familiares de vulnerabilidad económica y violencia doméstica, entre otras.

- Alta movilidad de niños y niñas; algunos de ellos recién arribados de departamentos del interior del país (migración interna).

- Maestra suplente proveniente de otro centro escolar recién integrada al plantel docente de esta escuela.

- Designación y asunción, por parte de niños y niñas, de roles estigmatizantes y marcados.

- Altos niveles de conflictividad relacional, bajos niveles de comunicación e incomprensión de las diferencias culturales.

- Incomprensión de la diversidad cultural expresada especialmente por los distintos estilos en el habla cotidiana ("vocabulario"); diferentes formas de organización familiar y del 
espacio doméstico; temporalidades distintas para la realización de tareas; actitudes de discriminación por características fenotípicas y/o de lugar de nacimiento y desconocimiento de las lógicas de la institución educativa y de la organización de la escuela en particular por parte de los adultos extranjeros referentes familiares recién arribados al país.

Teniendo presente estos planteamientos y basados en nuestras reflexiones, otra dificultad para acceder a sus derechos que detectamos en la población migrante al llegar a Uruguay (documentación —cédula identidad—, mercado laboral formal, vivienda, educación, entre otros), tuvo que ver con el desconocimiento respecto del acceso, uso y capacitación en Tecnologías de la Información y Comunicación (TIC). Esto se identificó tanto en la población adulta como en el sistema educativo, pero en este último caso de un modo más solapado. Durante el desarrollo de este proyecto observamos y analizamos algunas desventajas en los niños y niñas provenientes de países de la región que no accedieron al uso cotidiano de las TIC. En algunos países, el acceso a Internet es restringido, así como el uso de dispositivos digitales. Al llegar al sistema educativo uruguayo, su incorporación a las plataformas de aprendizaje del Proyecto de Conectividad Educativa para el Aprendizaje en Línea - Plan CElBAL (2010) no son inmediatas: algunos niños y niñas pasan meses sin poder acceder a las laptops. Cabe recordar que Uruguay hace más de 10 años que viene llevando adelante esta política de inclusión digital mediante la implementación de este Plan, el cual ha permitido el acceso a una laptop a todos los niños y niñas a nivel nacional, así como conectividad en todas las escuelas públicas del país. Es importante aclarar que dicho proyecto de extensión no se inscribe dentro del Plan CEIBAL pero, al trabajar en una escuela pública, se usó la disponibilidad tecnológica que los niños y niñas tenían en el aula, brindada por dicho Plan.

Nuestra intervención se sustentó desde una posición de "extensión crítica" (Tommasino y Cano, 2016) que deviene de los planteos de Paulo Freire. Por un lado, por el sentido del lugar asignado a los actores sociales con y entre los cuales se genera el conocimiento, no centrado exclusivamente en el saber del cual la academia es portadora sino situando a la misma como un actor más en el campo social en donde el saber se genera en un "entre" todos los participantes; en este caso, la escuela y los diversos actores educativos que se involucraron en el desarrollo del proyecto, los referentes adultos de los niños y niñas, los estudiantes universitarios y los propios niños y niñas en el ejercicio de su derecho a la participación. Por otro lado, coincidimos con el sentido ético-político que estos autores le atribuyen a las actividades de extensión en tanto compromiso académico con los problemas sociales que urgen a la sociedad de la cual la academia es parte. Asimismo, compartimos con esta perspectiva la importancia pedagógica que se le da a la extensión que, articulada con la formación, tiene un gran potencial educativo para los futuros profesionales, al tiempo que implica otra forma de hacer docencia; y por último, al lugar fundamental que ocupa como estrategia integral articulando, además de lo ya citado, la investigación con la enseñanza.

\section{Perspectiva teórica}

El sustento teórico de la propuesta retoma principios gestados en la Reforma Universitaria de 1918 en América Latina, que se inició en Córdoba, Argentina, donde la extensión universitaria ocupa un lugar central. Ello ha permitido históricamente generar en muchas univer- 
sidades del continente un compromiso ético-político y académico con las problemáticas sociales de los sectores más vulnerables de cada sociedad, así como la problematización teórica del concepto en sí, tal como lo hizo Paulo Freire en su libro ¿Extensión o comunicación? de 1971. Este concepto es polisémico, responde en parte a la heterogeneidad de actividades que comprende, y en parte a la perspectiva y al marco teórico desde el cual se parte. Emergen así términos tales como: articulación, vinculación con el medio, compromiso social universitario, extensión crítica, en donde prima, con diferentes matices, la visión de la extensión en tanto proceso pedagógico transformador para todos los actores involucrados.

Basados en esos principios apostamos en este trabajo por la complementariedad y el diálogo de aportes provenientes desde la Psicología Social Comunitaria, la Psicología Histórico-Cultural y una perspectiva crítica sobre los procesos de apropiación de las TIC.

La Psicología Social Comunitaria se enmarca en el paradigma de la construcción y transformación crítica. Montero (2004) desarrolla dicho paradigma mediante dimensiones fundamentales tales como la ontológica, epistemológica, metodológica, ética y política. Estos planteos dan soporte al trabajo de extensión desarrollado en el proyecto en el cual se trabajó para conformar un sistema de decisión compartida con los protagonistas, así como el interés por la transformación de la realidad identificada en el aula y sus dificultades para convivir desde las diferencias.

Dicha autora plantea que el aspecto ontológico hace referencia al carácter productor del conocimiento con alguien que construye realidad y protagoniza la vida cotidiana. En ese sentido, la transformación implicó cambiar la realidad del aula con el protagonismo de los familiares de los niños y niñas, la maestra y los propios niños y niñas participantes. En cuanto a la dimensión epistemológica, plantea que el conocimiento se produce siempre mediante las relaciones. El sujeto construye la realidad que a su vez lo transforma, lo limita y lo impulsa. La dimensión metodológica se relaciona con la capacidad de cambiar, transformar a partir de problemas concretos, incluyendo una estrategia dialógica, participativa y sustentada en una ética de la relación, con capacidad y derecho para decidir.

Para la Psicología Social Comunitaria, teoría y práctica no pueden separarse y es fundamental que los integrantes de las comunidades tengan derecho a tomar decisiones sobre aquellos asuntos que les conciernen. La dimensión política se entiende como acción transformadora, con carácter y finalidad del conocimiento producido, apostando por la construcción de lo público en aporte a políticas públicas específicas. Estos fundamentos guiaron el encuentro con los sujetos participantes de este proyecto y nos posibilitaron posicionarnos desde el comienzo en el diálogo, con respeto en cuanto a sus necesidades, promoviendo su participación y valorando sus saberes particulares. Desde este paradigma se cuestionan las jerarquías dominantes; por ello, se buscó impulsar una conciencia crítica en relación con el ejercicio de derechos, estimulando la participación de la población para incidir en la toma de decisiones. Se respetaron las historias, expectativas y las necesidades de la población a través de la reflexión permanente sobre el proceso que se iba dando y el sentido de lo que hacíamos, fomentando la participación. En este sentido, fueron claves la participación de la directora de la escuela, de la maestra del aula y de los familiares de los niños y niñas, los cuales se constituyeron en interlocutores durante todo el desarrollo del proyecto. En el trabajo concreto con los niños y niñas, estas dimensiones fueron fundamentales en la construcción de la propuesta, en la que, como se dijo, se invo- 
lucraron también estudiantes universitarios de la carrera de Psicología que acompañaron el trabajo en el aula.

Desde el enfoque teórico-metodológico de la Psicología Cultural son importantes el estudio del contexto donde los sujetos participan, el sentido, la construcción social de significados, así como la elaboración sobre la apropiación de los diferentes artefactos culturales en un momento histórico concreto: "la meta de la Psicología Cultural es entender cómo los procesos de desarrollo humano tienen lugar en la cultura" (De la Mata y Cubero, 2003:185).

Esta corriente de la psicología busca explicar las relaciones entre la acción humana, el funcionamiento mental, el desarrollo humano y las situaciones culturales, institucionales e históricas en las que se da ese funcionamiento (Wertsch, 1991). Parte de la idea de que la mente y la cultura se constituyen mutuamente y de que "la unidad de análisis es la vivencia o el modo cómo las personas valoran, perciben, interpretan aquello que les sucede y les rodea" (Esteban-Guitart, 2008:7). Michael Cole (1996) plantea que la cultura es un medio entretejido conjuntamente a través del cual se desenvuelve la vida humana. Esteban-Guitart lo sintetiza diciendo que "la cultura se entiende como símbolos compartidos, conceptos, significados, prácticas que definen y se generan a través de unidades culturales como la familia, el barrio, una comunidad o un país" (2008:10).

Otro concepto fundamental que destacamos desde esta perspectiva es el de Zona de Desarrollo Próximo (ZDP) de Vygotsky (1988). El mismo nos permite pensar en los procesos de construcción de conocimiento compartido en un grupo. El autor dirá que la ZDP es: "la distancia entre el nivel de desarrollo actual determinado por la solución independiente de un problema y el nivel de desarrollo potencial determinado mediante la solución de un problema bajo la guía de un adulto o la colaboración con iguales" (1988:87). Engeström (1987) considera la ZDP desde una dimensión colectiva, donde la distancia entre las acciones cotidianas de las personas y una nueva actividad social son gestadas a partir de procesos colectivos, haciendo foco en las transformaciones sociales.

Desde esta perspectiva teórica surge el Modelo colaborativo 5D, como ya mencionamos en la Introducción, que fue creado por Michael Cole (2006) a partir del trabajo con población migrante mexicana en Estados Unidos con la intención de generar procesos de inclusión educativa, social y cultural. El Modelo implica la construcción de significados compartidos, donde las TIC son mediadoras para la creación de configuraciones culturales en la cual los participantes se involucran naturalmente. El autor concibe a la 5D como una herramienta para la gestión de la diversidad y la inclusión social, con utilización de las TIC orientadas a metas significativas y construidas colectivamente. En Uruguay, este Modelo tiene dos antecedentes fundamentales, la investigación realizada en contextos comunitarios (Da Silva, 2018) y el trabajo en contextos de educación especial con niños y niñas con discapacidad intelectual y motriz (Viera, Da Silva, Falero y Moreira, 2014).

En lo que concierne a una perspectiva crítica de los procesos de apropiación de las TIC, sostenemos que estas abren oportunidades para el desarrollo a través de su utilización, pero también se sabe que se han generado nuevas desigualdades a partir de su existencia (Rivoir, 2009). Existen claras tendencias antagónicas en relación con las TIC; algunos las ven como la panacea que da solución al desarrollo de los países; otros, como un factor más que profundiza las desigualdades ya existentes. Sostenemos que las TIC no son en sí mismas ni responsables ni inocuas y que es importante atender a las intencionalidades 
con las que se incluyen en un proyecto. En este que abordamos se integraron en función de las necesidades del trabajo con los niños y niñas y el desarrollo de la actividad, con la intencionalidad de generar procesos de inclusión donde las TIC fueron pensadas como medios y no fines en sí mismas.

\section{Abordaje metodológico}

Se implementó una metodología cualitativa, con una estrategia de acción y colaboración de corte interdisciplinario para el abordaje de la problemática planteada en el proyecto: la movilidad humana y, específicamente, las temáticas referidas a la inclusión educativa de la niñez migrante en la escuela.

Esta elección epistémico-metodológica se fundamentó desde una visión dialógica, flexible, colaborativa, habilitante del análisis participativo de las necesidades y expectativas de los sujetos participantes, buscando producir conocimientos situados sobre los procesos de inclusión educativa, social y cultural de niños y niñas participantes, así como del grupo que conformaron los estudiantes, de modo de posibilitar también una evaluación continua de dichos procesos.

Se presenta a continuación una síntesis descriptiva de la propuesta desarrollada en el aula:

a) La propuesta basada en el Modelo colaborativo 5D se desarrolló mediante la construcción de un laberinto. Se diseñó teniendo en cuenta la temática de la convivencia intercultural y los procesos de movilidad humana. Por ello, se creó un personaje que fue el hilo conductor de la narrativa con la que trabajamos, se llamó Mago Movimiento. La narrativa se centraba en este Mago que viajaba por el mundo y que había llegado a la escuela para conocerlos y para pedir a los niños y niñas ayuda para recuperar su magia perdida. Esta magia se recuperaba con la ayuda de los niños y niñas que cumplían juntos ciertos desafíos de modo colaborativo para alcanzar diferentes metas. Ello involucró procesos comunicativos mediados por TIC, actividades lúdico-pedagógicas para el conocimiento entre los niños y niñas y sus respectivas culturas, así como desafíos cognitivos y expresiones afectivas sobre lo que estaban viviendo durante el proceso de trabajo.

Los principales recursos TIC utilizados fueron: una plataforma virtual Moodle (de uso específico para los proyectos de 5D Uruguay), notebooks y cámaras fotográficas (adquiridas para la realización del proyecto), tablets y servicio de conexión a Internet a través de disponibles en la escuela.

b) Guías de tareas con desafíos realizadas por los docentes con algún grado de colaboración de los estudiantes universitarios. En las mismas había misiones que apuntaban a la visibilización e intercambio cultural y al reconocimiento de las diferencias para establecer posibles conexiones parciales.

c) La maqueta fue la representación gráfica de aspectos de la narrativa y también representaba el laberinto y los posibles recorridos que podían realizar los niños y niñas.

d) La plataforma Moodle fue el espacio virtual donde se desarrolló la actividad y el principal canal de comunicación con el Mago Movimiento. Este espacio fue implementado y administrado por dos de los docentes universitarios integrantes del proyecto.

e) Se establecieron reglas de funcionamiento explícitas que tuvieron por objetivo organizar el conjunto de la actividad y que surgieron en el acuerdo de trabajo entre los niños, 
niñas, la maestra, los estudiantes y docentes universitarios. Cada niño y niña firmaba las reglas y se comprometía a cumplirlas.

f) El Mago Movimiento se comunicaba personalmente con cada uno de los niños y niñas mediante carta y/o foro de Moodle. Este personaje nunca se presentó fuera del mundo virtual.

g) Hubo una evaluación continua de la propuesta durante todo el proceso de trabajo con los participantes. Luego de cada encuentro de trabajo con los niños y niñas, se establecía una instancia de diálogo y evaluación entre los estudiantes universitarios, los docentes que acompañarban el proceso en el campo y la maestra de la escuela.

\section{Resultados y problematización de la experiencia de extensión}

Se presentan tres dimensiones problematizadoras construidas desde un proceso dialógi$\mathrm{co}$, con contenidos que respetan un modo "más performativo que representativo" (Sisto, 2008). Estas dimensiones atienden a los objetivos de la intervención y al proceso de trabajo desarrollado con la población. Se presentan las características del diseño colaborativo 5D logrado y los avatares para generar un aula intercultural; los aspectos vinculados al papel de la narrativa como hilo conductor de la propuesta y el lugar de las TIC como artefactos mediadores en el trabajo en el aula.

\section{1) Características del diseño 5D y la creación de un aula intercultural.}

La adaptación del Modelo 5D a la realidad del aula y construir un espacio de encuentro intercultural no fue un proceso lineal, implicó pausas, cambios estratégicos y análisis permanente del sentido de la intervención. El trabajo contempló analizar e incorporar mediante la reflexión las necesidades inferidas y sentidas, expectativas y fondos de conocimiento de los participantes. Requirió esfuerzo lograr entendimientos entre niños, niñas, estudiantes, docentes y familiares, a la vez que establecer límites a nuestra tarea de extensión, analizando nuestra propia implicación y posibilidades.

La construcción de confianza con la maestra y la directora de la escuela implicó desarrollar acciones que fueron estableciendo niveles de sintonía con relación al trabajo que se desarrollaba. Poner en práctica un "trueque constructivo" (León, 2010) con la escuela necesitó demostrar que el intercambio tenía beneficios mutuos: por un lado, la formación de las y los estudiantes universitarios, por otro, la ayuda para la escuela en la resolución de conflictos dada la diversidad en el aula, donde tratamos de fortalecer la red afectiva existente y en construcción.

Respetamos e integramos las sugerencias de los referentes familiares, la docente y la Dirección de la escuela, pero problematizamos el pedido de trabajo, entendiendo que nuestra presencia suponía construir una demanda de trabajo que incluyera la voz de los niños y niñas en el aula. Se generaron procesos individuales y grupales de trabajo, atento a la agrupación de los niños y niñas por afinidad, para luego crear espacios de conexiones parciales y producir prácticas de inclusión del "otro/a", habilitando a la escucha, el compartir, a interacciones colaborativas y a la creación de productos compartidos.

Esto significó procesos fermentales en los diferentes momentos del proyecto de extensión. Con respecto a las y los estudiantes universitarios, los vinculó y comprometió con 
una situación considerada como problemática social actual; la praxis tensionó sus saberes teórico-metodológicos y los motivó a investigar sobre temáticas innovadoras que antes desconocían; los habilitó a participar de una modalidad pedagógica, horizontal y dialógica: enseñar, acompañar y aprehender; generó grupalidad entre pares y profundos intercambios; les posibilitó aprender a utilizar, en muchos casos, por vez primera, herramientas y técnicas de investigación y extensión, una actitud flexible e interrogativa en el campo y un compromiso y solidaridad muy profundos con los niños y niñas con los cuales trabajaron, al tiempo que los indujo a la reflexividad sobre sí y sus implicaciones en el campo. Simultáneamente, en algunos casos, amplió la visión sobre su rol profesional al aplicar sus saberes en campos antes impensados. En sus propias palabras:

"En lo personal, fue una experiencia que brindó la oportunidad de enriquecimiento no solo en lo académico sino también en lo personal y soy de la idea de que lo teórico no hay mejor forma de comprenderlo y aprehenderlo que a través de la práctica, donde la contingencia obliga a ponernos a prueba, a desplegar la creatividad, los recursos, el conocimiento. Leer experiencias y artículos sin el espacio de la escuela, sin haber sido partícipe de esta experiencia no hubiese sido lo mismo", (extracto Diario de Campo, A.C., 6/12/2018)

Las prácticas, normas y saberes estratégicos de las niñas y niños migrantes fueron valorados e integrados en la propuesta de manera de enriquecer el contexto escolar desde una perspectiva de respeto a las diferencias. En los diferentes desafíos se buscó la visibilización de la diversidad cultural en el aula. Para avanzar en el juego se hicieron actividades en las que los niños y niñas necesitaban informarse sobre características y prácticas de diferentes nacionalidades, como Cuba, Venezuela, República Dominicana y Perú. Lo que requería conocer sobre el país y escuchar a las niñas y niños de la clase de esas procedencias para avanzar en el juego. En ello, fueron claves la utilización didáctica de mapas, las narrativas orales y las escritas a través de la creación colaborativa de cuentos, la expresión plástica, el compartir diversos estilos musicales, canciones y bailes, la realización de videos, todo esto llevado a cabo por parte de los niños y niñas motivados tanto por el equipo docente y estudiantes universitarios así como por iniciativa propia.

Cabe aclarar que la adaptación del 5D en el aula implicó el trabajo en subgrupos de niñas y niños por afinidad, con el acompañamiento de uno o dos estudiantes universitarios de referencia permanente para las actividades y desafíos que emprendían. El rol del estudiante era de facilitador y acompañante durante el proceso de trabajo, en ningún momento debía dar instrucciones, posicionarse como experto, ni liderar la tarea. En ocasiones, ocupaban un rol de mediadores interculturales, como promotores del acercamiento de niños y niñas de diferentes culturas, para propiciar una actitud positiva y respetuosa de la diversidad, ayudar en la construcción de encuentros donde se expresaban las diferencias, y superar rechazos o exclusión cultural. Esto supuso conocer a los niños y niñas, calibrar sus posibilidades y motivarlos a resolver las actividades propuestas en el 5D mediante la proporción de ayudas, ser asertivos con el tipo de soporte que se les brindaba, e intentar tener un apoyo progresivo en función del dominio que iban logrando.

La aplicación de la Escala de Harter (1981) y de la Escala Sistémica de Observación de Clases (Baeza, 2009) contribuyó a una lectura compleja, dinámica y multidimensional en la 
comprensión de las dinámicas de interacción y los procesos de motivación que se despliegan en un entorno de aprendizaje colaborativo. Estos instrumentos fueron insumos importantes para diseñar estrategias específicas para el proceso de trabajo con los niños y niñas del aula.

La primera toma de esta Escala Harter (1981) permitió tener un panorama de la situación motivacional de los niños y niñas. Ayudó a identificar en algunos de ellos una orientación motivacional de predominio extrínseca y así generar propuestas en la actividad que atendieran al fortalecimiento de la autonomía.

Entre la primera toma y la segunda de la Escala (la primera antes de iniciar la actividad con los niños y niñas, y la segunda al finalizar el trabajo) no se observaron diferencias significativas a nivel grupal. Lo que sí se advirtió fue que se logró mantener una orientación motivacional intrínseca, sobre todo un incremento de lo intrínseco relativo al dominio dependiente versus el dominio independiente del maestro/adulto. Este aspecto es muy importante dado que la literatura demuestra que, a medida que los niños y niñas avanzan en el grado escolar, su motivación intrínseca comienza a disminuir, primando así una orientación de tipo extrínseca. Para la construcción de un aula de respeto a las diferencias culturales, la motivación intrínseca es fundamental, ya que su predominio refiere a la percepción positiva de los niños y niñas de las tareas, al criterio de evaluación interna, al dominio independiente y al interés personal. Estos aspectos se alejan de la necesidad de aprobación de la mirada externa y del involucramiento por recompensa. La motivación intrínseca aportó a la dimensión de la interculturalidad ya que implicó establecer lazos entre la cultura propia y la de otros, donde fue necesario la independencia y la reafirmación interna para la construcción de una transacción o contexto de encuentro. Ello requirió desarrollar la creatividad y generar procesos promotores de la autonomía de los niños y niñas.

En cuanto a la Escala Sistémica de Observación de Clases (ESOC) de Baeza (2009), se analizaron la cohesión, la flexibilidad y la comunicación. Se invitó a la maestra del aula a asumir el rol de observadora de las dinámicas subgrupales de trabajo entre los estudiantes y los niños y niñas. Muchas veces, ante intervenciones en contexto de aula, las maestras "aprovecharon" a realizar otras tareas y no logrando encontrar un lugar en dichos proyectos. Esto fue una preocupación permanente desde el equipo de investigación y una explicitación hacia la maestra, aclarando la importancia de su involucramiento. De esta manera, tuvo un lugar activo y participativo en el 5D, a la vez que aportó insumos interesantes en lo referente al desarrollo de la experiencia y a la dinámica de funcionamiento de los cuatro subgrupos armados entre niños, niñas y estudiantes en el aula.

De los resultados obtenidos se desprende lo siguiente: a) los cuatro subgrupos tuvieron un funcionamiento balanceado de tipo estructurado-conectado. Este funcionamiento se caracteriza por una dinámica donde existen roles y reglas claras combinados con una dinámica en la que los miembros se apoyan y ayudan entre sí, la motivación es mutua y cooperación y habilidad para compartir están presentes de forma frecuente; b) un alto nivel de cohesión y niveles medios de flexibilidad-comunicación; c) los registros cualitativos se corresponden con la puntuación en la Escala Likert. Es importante señalar que las observaciones realizadas resultan acotadas para dar información más profunda sobre el funcionamiento del trabajo en el Modelo 5D. Para el uso de este instrumento se sugiere la realización de 3 a 5 observaciones en cada grupo, por lo que se necesitan para una futura experiencia de similares características entre 12 y 20 observaciones. Asimismo, la posibilidad de que participen 2 ob- 
servadores permitiría realizar un acuerdo interjueces y ofrecer mayor información en el análisis de la información, en especial en lo que respecta a las dinámicas de diversidad cultural.

En cuanto al armado de la propuesta, se entendió el proceso de adaptación como la creación de un "tercer objeto" (Engeström, 2001) que implicó la capacidad de construir nuevos aprendizajes y transformaciones a partir de la adopción de un nuevo elemento. La propuesta se armó teniendo en cuenta las dificultades presentadas en el aula (falta de tolerancia, respeto, aceptación de las diferencias, discriminación), pero la estrategia no fue ir al choque de esas dificultades, sino que se optó por trabajar desde la creatividad, la narratividad, el juego y la vivencia, que son los canales naturales de expresión y conexión con los niños y niñas. Esto es un elemento que destacamos, pues para pensar en procesos interculturales se deben visibilizar las diferencias pero también establecer las conexiones y sintonías para la convivencia. Ello en tanto se asume que la diferencia nos enfrenta a nuestras propias dificultades de entendimiento del "otro" pero también nos debe habilitar a crear posibles caminos de encuentros.

Para cerrar esta dimensión de análisis, se destaca que las características de implementación del 5D permitió mejorar la comprensión de las diferencias tanto entre niños y niñas extranjeros y uruguayos, así como de la diversidad de situaciones existentes entre los niños y niñas uruguayos (institucionalizados, niño con diagnóstico de autismo, situaciones de vulnerabilidad social, afectiva y económica, ritmos de aprendizajes diferentes, niños y niñas provenientes de hogares de clase media, entre otros). A la vez, en el equipo de estudiantes y docentes se requirió trabajar las propias diferencias y el establecimiento de acuerdos en la práctica. No existía una mirada única sobre la niñez, sobre los procedimientos a desarrollar, ni tampoco sobre las diferencias en el aula. La formación en psicología muchas veces puede llevar a construir una mirada psicopatológica de muchos comportamientos de niños y niñas. La práctica de extensión fue una oportunidad para deconstruir elaboraciones preestablecidas sobre la niñez y las diferencias que se construyen. Por ejemplo, la categoría de niño o niña con autismo, sus posibilidades o restricciones se alteraron en el encuentro específico, dado que las singularidades desbordaron la delimitación de teorías o manuales.

"Cuando comencé a trabajar con M —niño con espectro autista- me sentía como teniendo que improvisar y esperaba a veces una guía de cómo hacerlo que no veía. Luego comprendí que esto formaba parte de mis propias inseguridades y lo mejor en realidad era tener esa autonomía de poder trabajar a mi ritmo y como se fuera dando, esto me llevó mucho tiempo de investigación sobre la enfermedad por fuera de las reuniones o las visitas a la escuela, pero me dieron la posibilidad de desenvolverme de una forma más independiente, condición necesaria para el desarrollo de nuestra profesión y sabiendo que si lo necesitaba el apoyo siempre estaba al alcance de la mano". (extracto Diario de Campo, M.L., 29/11/2018)

También, ciertos prejuicios sobre las procedencias socioeconómicas de los niños y niñas y sus posibilidades de aprendizaje o cognitivas se pusieron en discusión. Algunos de los estudiantes universitarios, con formación en educación e inserción laboral en centros educativos privados, comentaban sobre el nivel académico y las exigencias de la maestra al ser una escuela pública. Elementos relevantes para la formación que tienen un anclaje singular para el análisis y la discusión cuando se ponen en juego en una práctica específica de extensión. 
De acuerdo con lo expresado por la maestra y por los familiares en la última reunión mantenida con ellos, también en los hogares se visualizaron cambios a través de diálogos en los cuales los niños y niñas problematizaron el tema del racismo desde nuevas perspectivas, se mostraron más interesados por sus compañeros de clase, más generosos, produciéndose transformaciones en la dimensión afectiva entre pares.

En el correr del trabajo se fue estableciendo un modo de estar juntos que dio cuenta del sentido de pertenencia y procesos de identidad grupal y habilitó progresivamente nuevas formas de comunicación y comportamientos no "marcados" por actitudes agresivas, estigmatizantes y discriminatorias. En la educación formal, si bien ha habido avances, falta mucho por hacer para que devenga en un sistema integrador, es decir, pluriétnico e intercultural, que incorpore los valores culturales y éticos, la diversidad lingüística y las historiografías de otras regiones y países y, de esta manera, brindar escucha y dar voz a las minorías silenciadas (Barrios, 2016).

Durante las entrevistas de evaluación con la directora de la escuela, esta manifestó que el proyecto resultó muy significativo, con importantes logros que se vieron reflejados en los comportamientos de los niños y niñas participantes. Se mencionaron aspectos de comunicación respetuosa, sentido de pertenencia, rendimiento escolar, buenos tratos entre pares. Destacó también la importancia del hecho de "acercar" a los referentes familiares y/o padres al centro para participar de instancias de diálogo en donde se tuvieron en cuenta sus perspectivas.

\section{2) Papel de la narrativa como hilo conductor en el Modelo 5D.}

Los aportes de Bruner (1990) sobre el lugar de la narrativa como modo de pensar, resolver problemas y organizar nuestra experiencia de vida, guiaron la construcción de la narrativa que fue el hilo conductor de la propuesta. El personaje Mago Movimiento y su historia con desafíos nos envolvieron a todos en una historia que fuimos viviendo, cambiando y que nos permitió construir significados de forma conjunta. La narración se unió con la acción, se organizó la experiencia y se habilitó la construcción de sentidos en el entramado que en el aula se desarrollaron. Mediante la narración fuimos organizando y comunicando nuestra experiencia, a la vez que la dotamos de sentidos. Esto se logró en función de las relaciones que construimos entre los niños, niñas, la maestra, familiares y los universitarios. La narrativa se enlazó a la necesidad de construir una convivencia de respeto de las diferencias y buscando alianzas, algunas duraderas, otras parciales en cuanto a la idea de movilidad. Un elemento de conexión fue identificar en nuestras trayectorias los procesos de movilidad. En los niños y niñas migrantes, en los provenientes de hogares que se trasladaban de un barrio a otro, en los que transitaron migración interna, en las y los universitarios, provenientes de distintos puntos del país, incluso en diferentes trayectorias para llegar a la universidad.

La historia lograda permitió tener un anclaje en cada uno de los participantes, una ilación con la experiencia en el aula. Seguidamente, se presenta la historia lograda del Mago Movimiento:

"Desde hace muchísimo tiempo y en diferentes lugares se sabe que existe un Mago que se llama 'Movimiento'. Recorre distintos barrios, ciudades, países, tantos que ya se siente que es parte de cada lugar a donde va. Siempre se detiene en algún lugar para divertirse junto a los niños y niñas y conocer las cosas que les gusta hacer cuando están juntos. Se dice que el Mago 'Movimiento' no puede quedarse quieto, se viste con ropas de distintos 
países, es muy curioso y va cambiando a medida que aprende cosas que los niños y niñas con las tecnologías le van compartiendo y enseñando.

Nadie sabe cómo es el Mago Movimiento, algunos dicen que le gusta mucho comunicarse con los niños y niñas, y siempre los invita a participar en el universo mágico de los juegos. Los que se han comunicado con él, cuentan que necesita ayuda para entender cómo se habla en cada ciudad y país, porque hay muchas palabras para decir las mismas cosas, jeso al Mago Movimiento le encanta y las pone en su universo! También le gusta conocer sobre la escuela, y que los niños y niñas le cuenten mediante los juegos y desafíos que les gusta hacer con sus compañeros y compañeras. Para todo esto se necesita mucha imaginación y ganas de pasarla bien haciendo nuevos amigos.

Los que quieran saber más, tienen que estar atentos a los mensajes y las formas de comunicarse del curioso Mago Movimiento.

Suerte y a disfrutar de la aventura".

Esta historia se leía a los niños y niñas al inicio de la actividad; previamente, se habían realizado algunas actividades recreativas de conocimiento y de explicitación de las dificultades de relacionamiento a partir de las diferencias culturales. Se habían organizado subgrupos de trabajo con la integración de estudiantes de referencia por grupo. Así, se les presentó la historia del Mago Movimiento y se inició la actividad que incluía la metáfora de un viaje laberinto, con estaciones y pasaportes. Iniciaron un recorrido en donde tenían que cumplir de forma grupal y negociada el camino que recorrían y los desafíos que iban resolviendo.

La estructura narrativa de la propuesta se constituyó en un espacio "transitorio" entre la actividad y las dificultades de inclusión intercultural en el aula. En la propuesta se combinaron la estructura narrativa y el trabajo colaborativo centrados en la toma de decisiones de forma argumentativa. El contenido narrativo estuvo presente en los juegos, en las cartas que se enviaban en cada actividad, en la historia del Mago y en el viaje imaginario que se realizó. Se destaca que la narrativa incluyó el reto de solucionar problemas reales, de experimentar en el encuentro soluciones a los desafíos que se proponían, integrando aspectos cognitivos y afectivos. Los elementos imaginativos fueron creando un ambiente de interés que, unido a la tarea colaborativa, integró en el aula una estructura de funcionamiento distinta de las tradicionales.

Es importante resaltar, dentro de la narrativa en esta actividad, un aspecto particular, su componente vivo, que involucró a los niños y niñas para participar y modificar la historia a partir de sus propias acciones. No entraremos en detalles sobre las actividades específicas ya que no es objeto de este escrito. Solo cabe mencionar que la actividad combinó elementos lúdicos temáticos, cuentos, música, dramatizaciones, y apuntó a la expresión emocional de los niños y niñas y a una estética con relación a la realidad vivida en el aula. También es importante destacar el lugar del Mago Movimiento y su conexión emocional con los niños y niñas. Ellos se vincularon con el Mago a partir de una invitación y un pedido de ayuda, ya que este necesitaba recuperar su magia perdida. Fue así que los niños y niñas se involucraron en acontecimientos de la historia del personaje, experimentando junto a sus compañeros los desafíos para ayudarlo.

La narrativa como hilo conductor de la propuesta permitió explorar la frontera entre lo imaginario y la realidad, propició un espacio para procesar entre niños, niñas y adultos las dificultades de encuentros desde las diferencias. Bruner (1997) sostiene que las acciones 
humanas son "actos de significados", y es mediante el significado que se relacionan el individuo y la cultura; los significados tienen sus orígenes en la cultura que se crea. Crear la propuesta de esta manera apuntó a la construcción de un "tercer espacio" de encuentro cultural e intergeneracional.

\section{3) Las TIC como artefactos mediadores de la propuesta.}

Las TIC se incluyeron en la propuesta en función de la narrativa, para dotar de sentido su utilización en cuanto a los objetivos, metas, y para la resolución de los desafíos que el Mago presentó. Se elaboró un espacio virtual mediante la plataforma Moodle en donde se alojaron las actividades con los desafíos que se debían resolver — las guías de tareas con las reglasy también se dispuso un espacio de mensajes y un chat en el cual los niños y niñas se podían comunicar con el Mago. Estaba consignado en las guías de tareas que, al terminar cada actividad se comunicaran con el Mago Movimiento, manifestaran cómo se habían sentido y qué logros o dificultades habían tenido. Al próximo encuentro recibían un feedback del Mago por medio de un mensaje en Moodle y también en un sobre con una carta en papel.

Se creó un usuario para cada niño y niña, con el cual accedían a la plataforma Moodle 5D Uruguay. La primera vez que ingresaron se encontraron con la página principal, en donde se visualizaba el sombrero del Mago Movimiento. La estética de la plataforma se ajustó para esta experiencia puntual y se pensó en la elaboración del sombrero como elemento distintivo del personaje en cuestión.

Mediante un desafío de acertijo inicial en el aula, con la ayuda de los estudiantes universitarios, descubrieron la clave de automatriculación (magia) que los conducía al espacio "Los viajes del Mago Movimiento", creado dentro la plataforma con el fin de llevar adelante la propuesta de "laberinto" en el marco del Modelo 5D.

Figura 1. Portada "Los viajes del Mago Movimiento" diseñada para la presente experiencia.

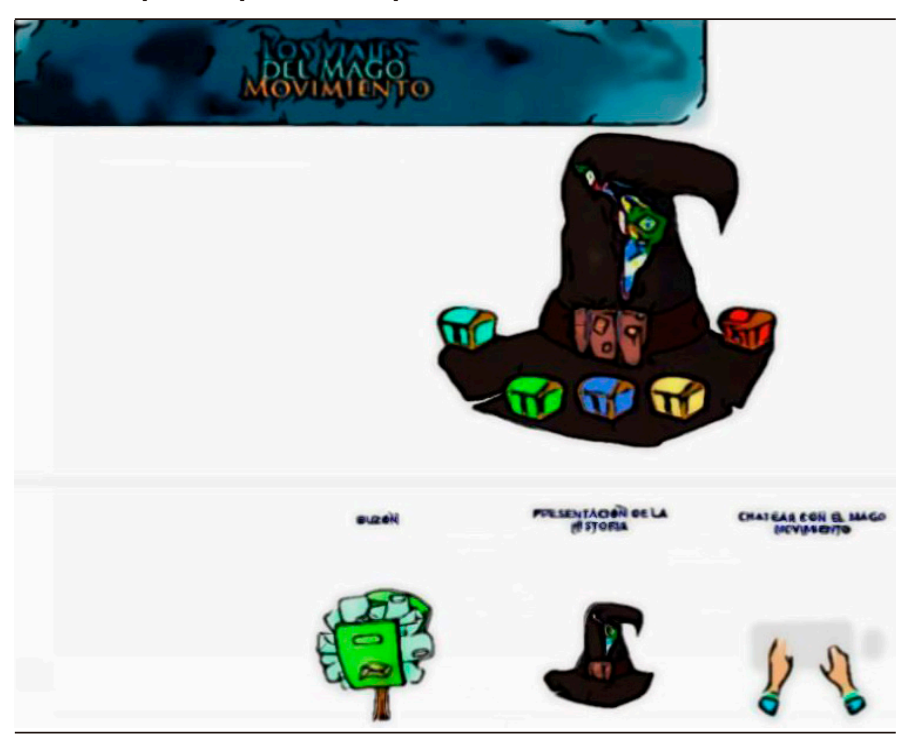

Fuente: elaboración propia, plataforma Moodle. 
Se consideró pertinente incluir el sombrero del Mago Movimiento en todas las actividades a lo largo del proceso. Por lo que sobre el mismo se agregaron diferentes cofres de colores, los cuales conforman el laberinto/recorrido por el cual los niños y niñas transitaron. Por otra parte, se editaron otras actividades y recursos para la interacción con los niños y niñas: un "foro" que ofició de buzón, una "página" para la historia del Mago Movimiento y un chat para conversar con el mismo.

El ícono "buzón" oficiaba como medio de comunicación asincrónico, dado que era un "Foro" dentro del espacio "Los viajes del Mago Movimiento", que fue editado con dicha finalidad. Aquí los niños y niñas escribían mensajes al Mago Movimiento, los cuales luego eran respondidos por los estudiantes universitarios con el fin de ir completando los diversos desafíos que se proponían durante toda la actividad de laberinto.

Para la presentación de la historia, como se dijo, se utilizó el sombrero del Mago Movimiento y fue enlazada con un recurso "Página" de la plataforma Moodle, en donde se agregó la historia escrita y además se enlazó igualmente la grabación de la misma en formato de audio mediante Google Drive.

En tanto, el tercer icono (las manos en el teclado), debajo del sombrero con los cofres, ofició como actividad chat editada en la plataforma Moodle y enlazada al mismo, la cual permitió realizar una sesión de conversación sincrónica con el Mago Movimiento (a cargo de un integrante del equipo docente), previo a la finalización del proceso.

Por su parte, cada uno de los cofres (ubicados encima del sombrero del Mago) corresponde a un curso dentro de la plataforma Moodle, con diferentes desafíos, que los niños y niñas debían cumplir para ayudar al Mago Movimiento a recuperar su magia perdida. La Tabla 1 presenta el nombre de la estación/cofre y la clave que debían descubrir para entrar. Una vez adentro, cumplían el desafío y luego le escribían al Mago.

Tabla 1. Estructura de las estaciones en la plataforma.

\begin{tabular}{|c|c|c|}
\hline Color del cofre & Tema de la estación & Clave de acertijo \\
\hline Celeste & Escuela & Dirección \\
\hline Verde & Sueños & Imaginación \\
\hline Amarillo & Encuentros & Abrazos \\
\hline Azul & Palabras & Escucha \\
\hline Rojo & Juegos & Aprender \\
\hline
\end{tabular}

Fuente: elaboración propia (2019).

Cada una de las estaciones/cofres alojaba su correspondiente color específico y, dentro de ellas, los desafíos a realizar. Todas estas estaciones/cofres fueron editadas ajustándose al diseño general pensado para la propuesta. Cuando los niños y niñas lograban acceder a uno de los cofres, a través de la clave de automatriculación, lo que hacían era ingresar al espacio creado en Moodle para tal fin. Posteriormente, haciendo clic en el cofre visualizaban un archivo de documentos de Google que contenía los desafíos propuestos por el 
Mago Movimiento. A modo de ejemplo, en la Figura 2 agregamos la imagen de la estación "Escuela" —cofre celeste-

Figura 2. Cofre "Escuela" creado y diseñado en plataforma Moodle para la presente experiencia.
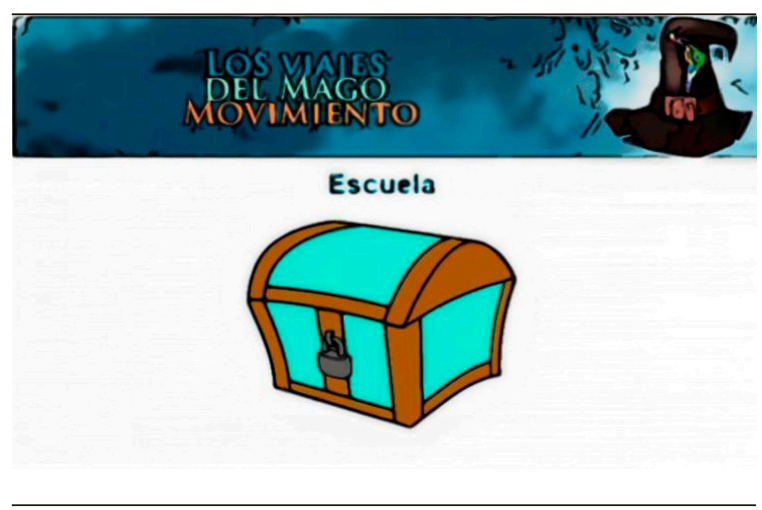

Fuente: elaboración propia.

En cuanto al trabajo en clase con los niños y niñas vinculado al uso de las TIC, se usaron tablets y computadoras proporcionadas por el equipo de investigación que se disponían para el trabajo en los subgrupos. Al ser usadas en una actividad nueva para todos los niños y niñas, posibilitaron establecer un piso común, un inicio similar para todos y todas. De esta manera, a los niños y niñas migrantes con menor uso de artefactos tecnológicos los ubicó en similares condiciones que el resto de sus compañeros. También el acompañamiento de estudiantes y el trabajo colaborativo con pares permitieron establecer un vínculo con la tecnología diferente de lógicas individuales y competitivas. Las TIC fueron usadas de forma natural por los niños y niñas para comunicarse, colaborar, buscar información, jugar y avanzar en la propuesta del Mago Movimiento, y no necesitaron recibir una instrucción ni formación instrumental específica sobre el uso. Avanzaban en complejidad a partir del acompañamiento de los estudiantes universitarios y el propio interés que la propuesta les provocaba. La dimensión narrativa de dicha propuesta también tuvo su soporte en las TIC, en el espacio virtual logrado, en la estética construida y en la motivación permanente para la construcción de una estructura donde la escritura fue fundamental mediante la correspondencia entre el Mago y los niños y niñas.

El empleo de tecnologías fue pensado desde la esfera de los procesos de apropiación colaborativa, donde la negociación, el compartir y tener una utilidad con sentido se acordaron como estrategia para su uso. Esto supuso un trabajo arduo, ya que las lógicas de usos individualizadas y centradas en videojuegos se colaban permanentemente. Pero a medida que se iba profundizando en el vínculo entre los niños, niñas y los estudiantes universitarios, aumentaron el compromiso, el sentido de pertenencia, la participación en la actividad, y el uso colaborativo de las TIC disponibles. 


\section{Consideraciones finales}

La experiencia de extensión desde la que reflexionamos en este artículo nos ha permitido prestar atención a las relaciones de reciprocidad entre la academia y la comunidad educativa. En las líneas planteadas se ha buscado profundizar en el conocimiento "entre" los participantes sobre un tema que hoy es prioritario en la agenda pública de nuestro país: la movilidad humana y específicamente la niñez migrante, de manera de cumplir con la dimensión ético-política de la extensión mediante el compromiso de trabajar en situaciones de relevancia social.

Las dimensiones esbozadas precedentemente nos permiten arribar a algunas reflexiones sobre el papel de la construcción de propuestas situadas, basadas en la identificación de las dificultades en un aula para la convivencia desde la diversidad y el respeto de las singularidades de procedencias. Esto no solo afectaba a la población migrante sino también a niños y niñas que, por diferentes razones (sociales, económicas y de salud), no sentían a la escuela como un lugar de referencia. Por ello la propuesta buscó construir una narrativa que apuntó a generar colaboración en función de metas, promovió pertenencia, generación de vivencias significativas y el uso de TIC en función de metas.

Trabajar para la construcción de una propuesta inclusiva requirió establecer mutualidades que se alejaron de construcciones compensatorias o ideas centradas en ubicar a los migrantes como sujetos carentes. La mirada estuvo focalizada en una postura que los ubicó como sujetos de derecho, constructores de ciudadanía y de una convivencia articulada desde las diferencias. Construir una propuesta basada en la colaboración implicó mirar desde las capacidades y los intercambios posibles, lo que significó trabajar para un diálogo desde una posición de mutua legitimidad y reconocimiento, considerando a los niños y niñas sujetos con agencia propia y no objetos.

El Modelo 5D fue adaptado al contexto específico del aula a través de la construcción de la narrativa del Mago Movimiento, pensado como un tercer espacio de convergencia de intereses en proceso, donde se fue configurando una nueva manera de estar juntos. Donde reconocemos las diferencias pero no para alejarnos o discriminarnos, sino para ver qué podemos hacer con ellas, qué nos potencia en el encuentro, cómo nos nutrimos en los intercambios y, en definitiva, cómo convivimos y aprendemos en el aula.

La apuesta por la construcción de una actividad de estas características implicó, por un lado, construir un modo empírico-investigativo comprometido con la transformación; por otro lado, intentar develar actitudes de invisibilización y naturalización de la discriminación. Lo cual se aleja de procesos idealizados, ya que requirió de muchos esfuerzos para converger y habitar un espacio compartido, reconocer las diferencias y aceptar que, más allá de estas, es posible construir intercambios en el supuesto de que la convivencia intercultural enriquece a todos quienes participan de ella, brindando una amplitud de alternativas antes desconocida. Estos procesos son situados, parciales, basados en trueques constructivos, y buscaron fortalecer la red afectiva y cognitiva de los niños y niñas en el aula (León, 2010).

Los fondos de conocimientos de las familias migrantes se intentaron integrar a las actividades de manera de romper algunas fronteras y construir una comunidad nueva o ampliada, donde aprender a respetar "lo distinto" fue unos de los focos para propiciar encuentros sostenidos desde la reciprocidad auténtica. Por ejemplo, la importancia de la música, el sentido de la demostración de afecto, la contextualización de palabras, la significación del apoyo de 
las personas adultas, la importancia de la espiritualidad, e incluso el valor de algunos alimentos, son esenciales en algunas tradiciones culturales. Estos elementos incluidos en actividades pertinentes, trascendiendo su aspecto "folclórico", pueden revelar, a modo de reflejo, aspectos poco analizados y visibilizados de las estructuras escolares y sus modos de funcionamiento. $Y$ esto nos interroga con relación al propio término interculturalidad y al cuestionamiento de si las diferencias son tantas y tan profundas. Nuestra experiencia nos brinda información empírica sobre las diferencias que van más allá de las trayectorias migratorias. La discriminación nos habla de un registro hegemónico o de códigos que se reproducen en la escuela respecto de una identidad nacional. Sabemos que esta experiencia es acotada y no transforma registros profundos de discriminación o estigmatizaciones culturales, pero sí abre un espacio de expresión y de interrogación sobre nuestras inscripciones hegemónicas en cuanto la escuela y la gestión de la diversidad.

Creemos que cultivar el sentimiento de pertenencia en el aula, construir confianza y lazos afectivos, es una apuesta ético-política en la que la escuela debe involucrarse permanentemente, no solo por temas de movilidad humana, ya que esto no beneficia únicamente a niños y niñas migrantes. Es un beneficio para todos y todas quienes son parte de la vida escolar, ya que la exclusión permanente de los circuitos de socialización genera sentimientos de rechazo y aislamiento que llevan a muchos niños y niñas a transitar y/o abandonar espacios de socialización sin que estos tengan un sentido con relación a sus intereses, expectativas y deseos.

La propuesta lograda fue una oportunidad de inclusión y de aprendizaje para los estudiantes universitarios en el sentido Freiriano de "pedagogía de la transformación", en tanto les permitió revisar el contraste entre elementos teóricos y la práctica llevada adelante; fue un espacio valorado como una nueva forma de aprender y ejercer su rol como futuros profesionales en una propuesta concreta que les brindó la posibilidad de interrogarse y pensar nuevas iniciativas para futuros proyectos de extensión e investigación, así como descubrir nuevos ámbitos y campos temáticos de ejercicio profesional. Para los niños y niñas que iniciaban un vínculo con la escuela y también para los que estaban en el margen de ella, la propuesta buscó habilitar sentidos que permitieran la existencia desde la diversidad en el aula. Hasta el momento, podemos decir que fue una experiencia acotada y que es necesario mancomunar esfuerzos para construir antecedentes respecto de la creación de la escuela como un espacio de cuidado, promotor del desarrollo y afirmador de la multiplicidad de identidades, necesario para cultivar el sentimiento de pertenencia y confianza. Promover el desarrollo de propuestas orientadas a prevenir la discriminación debido a las diferencias identitarias —en su más amplio sentido-y establecer interacciones respetuosas y acciones transformadoras.

\section{Referencias bibliográficas}

Baeza, S. (2009). Funcionamiento y clima sociorrelacional del aula. Buenos Aires: Aprendizaje Hoy.

Barrios, G. (2012). El tratamiento de la diversidad lingüística en la educación uruguaya (2006-2008). Letras (Santa María), (enero/junio), 21, 42. En Sturza, E. y Celada, M.T. (Orgs.). Políticas lingüísticas: espaços, questões e agendas (pp. 15-44). 
Bruner, J. (1997). La educación, puerta de la cultura. Madrid: Aprendizaje Visor.

(2000 [1990]). Actos de significado. Más allá de la revolución cognitiva. Madrid: Alianza.

Cole, M. (1996). Cultural psychology: A once and future discipline. Cambridge: Harvard University Press.

Cole, M. and The Distributed Literacy Consortium (2006). The Fifth Dimension. New York: Russell Sage Foundation.

Da Silva, M. (2018). Apropiación de las TIC en la infancia y su impacto en la comunidad: posibilidades y límites del modelo educativo Quinta Dimensión en un contexto de vulnerabilidad social en Uruguay. Tesis doctoral en Educación y TIC. Universitat Oberta de Catalunya.

Da Silva, M.; Rivero, E. (2018). Proyecto: Construyendo ciudadanía digital mediante la creación de un espacio intercultural en la Ciudad Vieja de Montevideo. Comisión Sectorial de Extensión y Actividades en el Medio de la Universidad de la República Oriental del Uruguay.

Debandi, N. (16 de noviembre de 2018). Mesa de Diálogo Movilidad humana: desafíos en la agenda de derechos. Montevideo: UdelaR, Cátedra UNESCO de Derechos Humanos.

De la Mata, M.L. y Cubero, M. (2003). Cultural Psychology: Approaches to the study of the relationship between mind and culture. Infancia y Aprendizaje, 26(2), 181-199.

Engeström, Y. (1987). Learning by expanding: An activity-theoretical approach to developmental research. Helsinki: Orienta-Konsultit Oy.

(2001). Expansive learning at work: toward an activity theoretical reconceptualización. Journal of Education at Work, 14(1), 133-156.

Esteban-Guitart, M. (2008). Hacia una psicología cultural. Origen, desarrollo y perspectivas. Fundamentos en Humanidades, 9(18), 7-23.

Harter, S. (1981). A New Self-Report Scale of Intrinsic Versus Extrinsic Orientation in the Classroom: Motivational and Informational Components. Developmental Psychology, 17(3), 300-312.

Koolhaas, M.; Prieto, V.; Robaina, S. (2017). Informe final: Caracterización de las nuevas corrientes migratorias en Uruguay. Nuevos orígenes latinoamericanos: estudio de caso de las personas peruanas y dominicanas. Recuperado de: http://www.mides.gub.uy/innovaportal/file/75559/1/investigacion_caracterizacion2017_final_digital.pdf

León, A. (2010). Danzando la Psicología Social Comunitaria: revisitando la IAP a partir de un curso de danza en una asociación cultural de barrio. Athenea Digital, (17), 255-270.

Montero, M. (2004). Introducción a la Psicología Comunitaria. Desarrollo, conceptos y procesos. Buenos Aires: Paidós.

Moll, L.C. (1997). Vygotsky, la educaciòn y la cultura en acción. En Álvarez, A. (Ed.). Hacia un curriculum cultural. La vigencia de Vygotski en la educación (pp. 39-53). Madrid: Fundación Infancia y Aprendizaje.

Plan CEIBAL (2010). Informe de monitoreo y evaluación de impacto social del Plan Ceibal. Resumen Ejecutivo 2010. El Plan Ceibal a 2010: avances y desafíos. Recuperado de http://www.ceibal.edu.uy/es

Rivoir, A. L. (2009). Innovación para la inclusión digital. El Plan Ceibal en Uruguay. Mediaciones sociales. Revista de Ciencias Sociales y de la Comunicación, 4, 299-328.

Sisto, V. (2008). La investigación como una aventura de producción dialógica: La relación con el otro y los criterios de validación en la metodología cualitativa contemporánea. Psicoperspectivas, 7, 114-116.

Tommasino, H.; Cano, A. (2016). Modelos de extensión universitaria en las universidades latinoamericanas en el S XXI: tendencias y controversias. Universidades, (67), 7-23.

Uriarte, P.; Urruzola, J. (2018). Las mujeres, los niños y las niñas también migran. Corrientes migratorias latinoamericanas en Uruguay desde una perspectiva de género. Encuentros Latinoamericanos, III(2), 23-48. 
Viera, A.; Da Silva, M.; Falero, B. y Moreira, K. (2014). La Quinta Dimensión en el contexto de la Educación Especial en Uruguay. Ponencia en UC-Links Annual Conference. Universidad de California.

Vygotsky, L.S. (1988). El desarrollo de los procesos psicológicos superiores. Madrid: Grijalbo.

Walsh, C. (2009). Interculturalidad crítica y educación intercultural. Ponencia en Seminario "Interculturalidad y Educación Intercultural”. Instituto Internacional de Integración del Convenio Andrés Bello, La Paz, Bolivia.

Wertsch, J.V. (1991). Voices of the mind. London: Harvester Wheatsheaf. 